\title{
Extreme Voting under Proportional Representation: The Multidimensional Case
}

Francesco De Sinopoli and Giovanna Iannantuoni

$$
\text { June } 2005
$$

CWPE 0531

Not to be quoted without permission 


\title{
Extreme Voting under Proportional Representation: The Multidimensional Case
}

\author{
Francesco De Sinopoli*and Giovanna Iannantuoni ${ }^{\ddagger}$
}

March 10, 2005

\begin{abstract}
We study the strategic behavior of voters in a model of proportional representation, in which the policy space is multidimensional. Our main finding is that in large electorate, under some assumptions on voters' preferences, voters essentially vote, in any equilibrium, only for the extreme parties.

Keywords: Strategic Voting, Proportional Rule, Nash Equilibrium.

JEL Classification: C72, D72.
\end{abstract}

\section{Introduction}

In this short paper we examine the equilibria of a spatial model of proportional representation, in which the policy space is multidimensional and the policy outcome is a linear combination of parties' positions weighted by the share of votes each party gets in the election. The understanding of such issues is a fundamental step in order to study the relation between strategic voting and the number of parties resulting at equilibrium, as well as the relation between strategic voting and the position of the parties voters decide to vote for.

In a recent paper (De Sinopoli and Iannantuoni, 2003) we analyze a similar model of proportional representation, in which the policy space is unidimensional. The main result is that, if voters' preferences are single peaked, essentially an unique Nash equilibrium exists, characterized by the fact that any voter on the left/right of the corresponding policy outcome votes for the leftmost/rightmost party. The incentive to vote for an extreme is given by the maximal effect that such a vote has on the outcome.

In this paper, we show how this result extends to a multidimensional policy space. If we assume that the policy space is the unit square and there are parties located at the four corners, it is unambiguous that these parties are the extreme ones. In this political scenario, we prove that, if voters' preferences are single peaked in each dimension with the peak independent from the other dimension, only the extreme parties take a relevant amount of votes. Hence, under the above assumptions, the extreme result holds also when strategic voters face a two-dimensional policy space. However, a simple example shows that the assumption of strict quasi-concavity of the utility function, which is the natural extension of single-peakedness to the multidimensional case, is not sufficient to obtain the result that only the extreme parties get votes.

The hypothesis that four parties located at the corners exist can be relaxed, at the cost of dealing with a specific utility function. We study the case in which each voter has a loss function given by a weighted sum of each issue's distance from his preferred policy. In this circumstance, we demonstrate that only parties located on the boundary of the convex hull of parties' positions take a relevant amount of votes.

We emphasize that, even if all the results are proven assuming a two-dimensional policy space, the extensions to more than two dimensions are straightforward.

Before proceeding to the model, let us mention that Schofied and Sened (2002) present a model of multi-party spatial competition under proportional rule. Their main result, supported also by an empirical analysis to the Israeli politics, shows that "the centre is empty in politics".

\footnotetext{
*Department of Economics, Universidad Carlos III de Madrid. E-mail: fsinopol@eco.uc3m.es

${ }^{\dagger}$ Faculty of Economics, University of Cambridge. E-mail: gi209@cam.ac.uk

$\ddagger$ We want to thank Jean-François Mertens and Thomas Palfrey for precious discussions.
} 
We describe the model in the next Section, and, then, we study strategic voting when the four parties located at corners exist in Section 3, while in Section 4 we analyze the case in which each voter has a loss function given by a weighted sum of each issue's distance from his preferred policy. Section 5 concludes the paper. An appendix contains most of the proofs.

\section{The Model}

Policy Space. The policy space $\mathbb{X}$ is a rectangle and without loss of generality we assume $\mathbb{X}=[0,1]^{2}$.

Parties. Parties are fixed both in number and in their positions, in that there is no strategic role for them: there is an exogenously given set of parties $M=\{1, \ldots, k, \ldots m\}$, indexed by $k$. Each party $k$ is characterized by a policy $\zeta_{k}=\left(\zeta_{k 1}, \zeta_{k 2}\right) \in \mathbb{X}$.

Proportional Rule. Given the set of parties $M$, each voter can cast his vote for any party. ${ }^{1}$ The pure strategy space of each player $i$ is $S_{i}=\{1, \ldots, k, \ldots, m\}$ where each $k \in S_{i}$ is a vector of $m$ components with all zeros except for a one in position $k$, which represents the vote for party $k$.

A mixed strategy of player $i$ is a vector $\sigma_{i}=\left(\sigma_{i}^{1}, \ldots \sigma_{i}^{k}, \ldots, \sigma_{i}^{m}\right)$ where each $\sigma_{i}^{k}$ represents the probability that player $i$ votes for party $k$.

The policy outcome. The policy outcome is a linear combination of parties' policies each coefficient being equal to the corresponding share of votes. ${ }^{2}$ Given a pure strategy combination $s=\left(s_{1}, s_{2}, \ldots, s_{n}\right)$, $v(s)=\frac{1}{n} \sum_{i} s_{i}$ is the vector representing for each party its share of votes, hence the policy outcome can be written as:

$$
X(s)=\sum_{k=1}^{m} \zeta_{k} v_{k}(s) .
$$

Voters. Each voter $i$ is characterized by a bliss point $\theta_{i} \in \Theta=\Theta_{1} \times \Theta_{2}=[0,1]^{2}$. We assume that it exists a fundamental utility function (à la Harsanyi) $u: \Re^{4} \rightarrow \Re$, representing the preferences, which is $u_{i}(X)=u\left(X, \theta_{i}\right)$. In other words, a player is fully characterized by his bliss point.

Given the set of parties and the utility function $u$, a finite game $\Gamma$ is characterized by a set of players $N=\{1, \ldots, i, \ldots, n\}$ and their bliss points. Given $\Gamma$, let $F_{1}\left(\theta_{1}\right)$ and $F_{2}\left(\theta_{2}\right)$ be the marginal distributions of players' bliss points on $\Theta_{1}$ and $\Theta_{2}$, that is to say $F_{1}\left(\theta_{1}\right)$ is the proportion of voters $i$ with $\theta_{i 1} \leq \theta_{1}$, and $F_{2}\left(\theta_{2}\right)$ is the proportion of voters $i$ with $\theta_{i 2} \leq \theta_{2}$.

The utility that player $i$ gets under the strategy combination $s$ is:

$$
U_{i}(s)=u\left(X(s), \theta_{i}\right)
$$

Given a mixed strategy combination $\sigma=\left(\sigma_{1}, \ldots, \sigma_{n}\right)$, because players make their choice independently from each other, the probability that $s=\left(s_{1}, s_{2}, \ldots, s_{n}\right)$ occurs is:

$$
\sigma(s)=\prod_{i \in N} \sigma_{i}^{s_{i}} .
$$

The expected utility that player $i$ gets under the mixed strategy combination $\sigma$ is:

$$
U_{i}(\sigma)=\sum \sigma(s) U_{i}(s)
$$

In the following, as usual, we shall write $\sigma=\left(\sigma_{-i}, \sigma_{i}\right)$, where $\sigma_{-i}=\left(\sigma_{1}, \ldots \sigma_{i-1}, \sigma_{i+1}, \ldots \sigma_{n}\right)$ denotes the $(n-1)$-tuple of strategies of the players other than $i$. Furthermore, $s_{i}$ will denote the mixed strategy $\sigma_{i}$

\footnotetext{
${ }^{1}$ In this model we do not allow for abstention. We cannot claim that this assumption is neutral. In our proof we use the fact that, as the number of players goes to infinity, the weight of each player goes to zero, and this does not hold when a large number of voters abstains.

${ }^{2}$ Note that, if politicians have a loss function that is quadratic, our outcome function is the utilitarian solution of a bargaining process among them. Hence it is the result of a bargaining process of government formation à la Baron and Diermeier (2001), under the assumption that the statu quo is quite negative for parliamentary members. This is a weak assumption if the statu quo is given by new election where parliamentary members face the risk of not being reelected, and the cost of staying out of the legislature is sufficiently large, as in Austen-Smith and Banks (1988).
} 
that gives probability one to the pure strategy $s_{i}$. Given a mixed strategy combination $\sigma, \bar{\sigma}$ will denote the "average" strategy, i.e. $\bar{\sigma}=\sum_{i \in N} \frac{\sigma_{i}}{n}$, and, with abuse of notation, $X(\bar{\sigma})$ the corresponding outcome, that is $X(\bar{\sigma})=\sum_{k=1}^{m} \zeta_{k} \bar{\sigma}_{k}$

\section{Parties at the corners of the square}

In this section, we study the equilibria of the model when there are four parties located at the four corners of the policy space and voters' preferences are single peaked in $X_{1}\left(\right.$ resp. $X_{2}$ ) with the peak independent from $X_{2}\left(\right.$ resp. $\left.X_{1}\right)$. The two assumptions can be formulated as:

Assumption 1: The four extremist parties $L B, L T, R B, R T$ with $\zeta_{L B}=(0,0), \zeta_{L T}=(0,1), \zeta_{R B}=(1,0)$, $\zeta_{R T}=(1,1)$ exist. $^{3}$

Assumption 2:

$\forall X_{2}, \theta_{i}: X_{1}<X_{1}^{\prime} \leq \theta_{i 1}$ or $\theta_{i 1} \leq X_{1}^{\prime}<X_{1}$ implies $u\left(X_{1}, X_{2}, \theta_{i}\right)<u\left(X_{1}^{\prime}, X_{2}, \theta_{i}\right)$

$\forall X_{1}, \theta_{i}: X_{2}<X_{2}^{\prime} \leq \theta_{i 2}$ or $\theta_{i 2} \leq X_{2}^{\prime}<X_{2}$ implies $u\left(X_{1}, X_{2}, \theta_{i}\right)<u\left(X_{1}, X_{2}^{\prime}, \theta_{i}\right)$.

We first state a trivial result for pure strategies. Every pure strategy equilibrium is such that, except for voters located in a small subset of the policy space, whose area is inversely related to the number of players, voters vote only for the extreme parties.

Proposition 1 Under Assumptions 1 and 2, let $s$ be a pure strategy equilibrium of the game $\Gamma$ with $n$ voters, then

$(\alpha)$ if $\theta_{i} \leq X(s)-\frac{\overrightarrow{1}}{n}$ then $s_{i}=L B$

(B) if $\theta_{i} \geq X(s)+\frac{\overrightarrow{1}}{n}$ then $s_{i}=R T$;

$(\gamma)$ if $\theta_{i 1} \leq X_{1}(s)-\frac{1}{n}$ and $\theta_{i 2} \geq X_{2}(s)+\frac{1}{n}$ then $s_{i}=L T$;

( $\delta)$ if $\theta_{i 1} \geq X_{1}(s)+\frac{1}{n}$ and $\theta_{i 2} \leq X_{2}(s)-\frac{1}{n}$ then $s_{i}=R B$.

Proof. $(\alpha)$ First notice that if $\theta_{i} \leq X\left(s_{-i}, L B\right)$, then by Assumption 2 voting for $L B$ is the only best reply for player $i$ against $s_{-i}$. Because $X\left(s_{-i}, L B\right)=X(s)-\frac{1}{n} \zeta_{s_{i}} \geq X(s)-\frac{\overrightarrow{1}}{n}$, then $\theta_{i} \leq X(s)-\frac{\overrightarrow{1}}{n}$ implies that $L B$ is the unique best reply, for player $i$, to $s_{-i}$. $(\beta)(\gamma)(\delta)$ A symmetric argument holds.

\subsection{On the need of Assumption 2}

In De Sinopoli and Iannantuoni (2003) we have shown that single-peakedness of voters' preferences is the only assumption needed to prove that almost any voter, in any pure strategy equilibrium, votes only for the two extremist parties. Hence, it is quite natural to check if Assumption 2 can be relaxed by simply assuming that the voters' utility functions are strictly quasi-concave, that is the natural extension of singlepeakedness when the policy space is multidimensional. At this end, let us consider the following example with five parties, the four extremists $L B, L T, R B, R T$ (as described in Assumption 1) and the center $C$ with $\zeta_{c}=\left(\frac{1}{2}, \frac{1}{2}\right)$.

The strictly quasi-concave utility function of voter $i$, characterized by the bliss point $\theta_{i}=\left(\theta_{i 1}, \theta_{i 2}\right)$, is:

$$
u\left(X, \theta_{i}\right)=-\left(\sum_{j=1}^{2}\left(X_{j}-\theta_{i j}\right)^{2}\right)-10 \sqrt{\left[\left(\theta_{i 2}-\frac{1}{2}\right) X_{1}+\frac{1}{2}\left(\theta_{i 1}-\theta_{i 2}\right)-\left(\theta_{i 1}-\frac{1}{2}\right) X_{2}\right]^{2}} .
$$

Voters are located in four regions (see figure 1 ) on $\Theta=[0,1]^{2}$ :

\footnotetext{
${ }^{3}$ This Assumption could be weakened, at the cost of a much heavier notation, by simply requiring that the convex hull of parties' position is a rectangle, with the sides parallel to the axis.
} 


$$
\begin{aligned}
A & =\left\{\theta_{1} \leq \min \left\{\frac{3}{2} \theta_{2}-\frac{1}{4}, \frac{5}{4}-\frac{3}{2} \theta_{2}, \frac{1}{2}\right\}\right\} ; \\
B & =\left\{\theta_{1} \geq \max \left\{\frac{3}{2} \theta_{2}-\frac{1}{4}, \frac{5}{4}-\frac{3}{2} \theta_{2}, \frac{1}{2}\right\}\right\} ; \\
C & =\left\{\theta_{2} \leq \min \left\{\frac{3}{2} \theta_{1}-\frac{1}{4}, \frac{5}{4}-\frac{3}{2} \theta_{1}, \frac{1}{2}\right\}\right\} ; \\
D & =\left\{\theta_{2} \geq \max \left\{\frac{3}{2} \theta_{1}-\frac{1}{4}, \frac{5}{4}-\frac{3}{2} \theta_{1}, \frac{1}{2}\right\}\right\} .
\end{aligned}
$$

It takes few calculations to check that, independently from the number of voters, everybody voting for $C$ is a Nash Equilibrium of the game.

We show that for a player $i$ located in region $A$ and with $\theta_{i 2} \leq \frac{1}{2}$ (see figure 2), voting for the center is a best reply to everybody voting for the center. By symmetry, it will follow that everybody voting for the center is a Nash equilibrium of the game. Let $C_{-i}$ be the strategy combination given by everybody except player $i$ voting for $C$.

If player $i$ votes for $C$ the policy outcome is $\left(X_{1}, X_{2}\right)=\left(\frac{1}{2}, \frac{1}{2}\right)$, hence

$$
u\left(\left(C_{-i}, C\right), \theta_{i}\right)=-\left(\frac{1}{2}-\theta_{i 1}\right)^{2}-\left(\frac{1}{2}-\theta_{i 2}\right)^{2} .
$$

If player $i$ votes for $L B$ the policy outcome is $\left(X_{1}, X_{2}\right)=\left(\frac{1}{2}-\frac{1}{2 n}, \frac{1}{2}-\frac{1}{2 n}\right)$, hence $u\left(\left(C_{-i}, L B\right), \theta_{i}\right)=$ $-\left(\frac{1}{2}-\frac{1}{2 n}-\theta_{i 1}\right)^{2}-\left(\frac{1}{2}-\frac{1}{2 n}-\theta_{i 2}\right)^{2}-10 \sqrt{\left[\left(\theta_{i 2}-\frac{1}{2}\right)\left(\frac{1}{2}-\frac{1}{2 n}\right)+\frac{1}{2}\left(\theta_{i 1}-\theta_{i 2}\right)-\left(\theta_{i 1}-\frac{1}{2}\right)\left(\frac{1}{2}-\frac{1}{2 n}\right)\right]^{2}}$.

If player $i$ votes for $L T$ the policy outcome is $\left(X_{1}, X_{2}\right)=\left(\frac{1}{2}-\frac{1}{2 n}, \frac{1}{2}+\frac{1}{2 n}\right)$, hence $u\left(\left(C_{-i}, L T\right), \theta_{i}\right)=$ $-\left(\frac{1}{2}-\frac{1}{2 n}-\theta_{i 1}\right)^{2}-\left(\frac{1}{2}+\frac{1}{2 n}-\theta_{i 2}\right)^{2}-$ $-10 \sqrt{\left[\left(\theta_{i 2}-\frac{1}{2}\right)\left(\frac{1}{2}-\frac{1}{2 n}\right)+\frac{1}{2}\left(\theta_{i 1}-\theta_{i 2}\right)-\left(\theta_{i 1}-\frac{1}{2}\right)\left(\frac{1}{2}+\frac{1}{2 n}\right)\right]^{2}}$.

If player $i$ votes $R B$ the policy outcome is $\left(X_{1}, X_{2}\right)=\left(\frac{1}{2}+\frac{1}{2 n}, \frac{1}{2}-\frac{1}{2 n}\right)$, hence $u\left(\left(C_{-i}, R B\right), \theta_{i}\right)=$ $-\left(\frac{1}{2}+\frac{1}{2 n}-\theta_{i 1}\right)^{2}-\left(\frac{1}{2}-\frac{1}{2 n}-\theta_{i 2}\right)^{2}-10 \sqrt{\left[\left(\theta_{i 2}-\frac{1}{2}\right)\left(\frac{1}{2}+\frac{1}{2 n}\right)+\frac{1}{2}\left(\theta_{i 1}-\theta_{i 2}\right)-\left(\theta_{i 1}-\frac{1}{2}\right)\left(\frac{1}{2}-\frac{1}{2 n}\right)\right]^{2}}$.

If player $i$ votes $R T$ the policy outcome is $\left(X_{1}, X_{2}\right)=\left(\frac{1}{2}+\frac{1}{2 n}, \frac{1}{2}+\frac{1}{2 n}\right)$, hence $u\left(\left(C_{-i}, R T\right), \theta_{i}\right)=$ $-\left(\frac{1}{2}+\frac{1}{2 n}-\theta_{i 1}\right)^{2}-\left(\frac{1}{2}+\frac{1}{2 n}-\theta_{i 2}\right)^{2}-10 \sqrt{\left[\left(\theta_{i 2}-\frac{1}{2}\right)\left(\frac{1}{2}+\frac{1}{2 n}\right)+\frac{1}{2}\left(\theta_{i 1}-\theta_{i 2}\right)-\left(\theta_{i 1}-\frac{1}{2}\right)\left(\frac{1}{2}+\frac{1}{2 n}\right)\right]^{2}}$.

The first easy observation is that voting $L B$ is a better reply than voting for any other extremist party ${ }^{4}$.

Hence if $u\left(\left(C_{-i}, C\right), \theta_{i}\right) \geq u\left(\left(C_{-i}, L B\right), \theta_{i}\right)$ voting for $C$ is a best reply for player $i$.

It is easy to calculate that

$u\left(\left(C_{-i}, C\right), \theta_{i}\right)-u\left(\left(C_{-i}, L B\right), \theta_{i}\right)=\frac{1}{n}\left[\frac{1}{4 n}-1-4 \theta_{i 1}+6 \theta_{i 2}\right]$, which is strictly positive since $\theta_{i} \in A$ (i.e. $\left.\theta_{i 1} \leq \frac{3}{2} \theta_{i 2}-\frac{1}{4}\right)$.

By symmetry, everybody voting for $C$ is a Nash equilibrium (independently from the number of the players), and, hence, the assumption of strictly quasi-concavity of the utility function does not imply that voters vote only for the parties located at the corners.

${ }^{4}$ For example, $u\left(\left(C_{-i}, L B\right), \theta_{i}\right)-u\left(\left(C_{-i}, L T\right), \theta_{i}\right)=\frac{6}{n}\left(1-2 \theta_{i 2}\right)$ that is non-negative for $\theta_{i 2} \leq \frac{1}{2}$. 


\subsection{Mixed strategy equilibria}

In this section we analyze mixed strategy equilibria. To carry on such analysis we assume that the utility function is continuously differentiable respect to the policy. ${ }^{5}$

Assumption 3: The fundamental utility function $u: \Re^{4} \rightarrow \Re$ is continuously differentiable with respect to the policy. ${ }^{6}$

In view of the result of Proposition 1, it is natural to analyze the policy outcome obtained when anybody strictly to the left-bottom of it votes for $L B$, anybody strictly to the left-top votes for $L T$ and so on.

At this end, let $\theta^{\Gamma}=\left(\theta_{1}^{\Gamma}, \theta_{2}^{\Gamma}\right)$ be the unique fixed point of the correspondence $H: \Theta \rightarrow \Theta$ defined by: ${ }^{7}$

$$
\begin{aligned}
& H_{1}(\theta)=\left[1-F_{1}\left(\theta_{1}\right), \lim _{y \rightarrow \theta_{1}^{-}} 1-F_{1}\left(\theta_{1}\right)\right] \\
& H_{2}(\theta)=\left[1-F_{2}\left(\theta_{2}\right), \lim _{y \rightarrow \theta_{2}^{-}} 1-F_{2}\left(\theta_{2}\right)\right] .
\end{aligned}
$$

In the appendix we will prove the following results:

Proposition 2 Under assumptions 1, 2 and 3, $\forall \eta>0, \exists n_{0}$ such that $\forall n \geq n_{0}$ if $\sigma$ is a Nash equilibrium of a game $\Gamma$ with $n$ voters, then:

( $\alpha$ ) if $\theta_{i} \leq \theta^{\Gamma}-\vec{\eta}$ then $\sigma_{i}=L B$

( $\beta$ ) if $\theta_{i} \geq \theta^{\Gamma}+\vec{\eta}$ then $\sigma_{i}=R T$;

$(\gamma)$ if $\theta_{i 1} \leq \theta_{1}^{\Gamma}-\eta$ and $\theta_{i 2} \geq \theta_{2}^{\Gamma}+\eta$ then $\sigma_{i}=L T$;

( $\delta$ ) if $\theta_{i 1} \geq \theta_{1}^{\Gamma}+\eta$ and $\theta_{i 2} \leq \theta_{2}^{\Gamma}-\eta$ then $\sigma_{i}=R B$.

The above proposition replicates and extends the result previously obtained for pure strategy equilibria. It replicates the previous result in the sense that every equilibrium is such that, except for voters located in a small subset of the policy space, whose area is inversely related to the number of players, voters vote only for the extreme parties. It extends it, because it shows that every equilibrium conforms to the same "cutpoint" $\theta^{\Gamma}$.

\section{A specific utility function}

In the previous section we have made a very strong political assumption, that is four parties located at the corners of the policy space exist. In the following, we will drop Assumption 1, at the cost to deal with a specific utility function, which corresponds to a loss function equal to a weighted sum of each issue's distance from the preferred policy:

Assumption 4: The fundamental utility function $u: \Re^{4} \rightarrow \Re$ is:

$$
u\left(X, \theta_{i}\right)=-\alpha\left(\theta_{i}\right)\left|\theta_{i 1}-X_{1}\right|-\left|\theta_{i 2}-X_{2}\right|
$$

where $\alpha\left(\theta_{i}\right):[0,1]^{2} \rightarrow \Re_{++}$is a continuous function.

Also in this case, for large electorate, an "extreme" result holds. In the appendix, we will prove:

\footnotetext{
${ }^{5}$ To study mixed strategies equilibria some cardinal assumptions on the utility function are needed. Because we use the mean value theorem, the cardinal assumption we have made is the differentiability one. Furthermore, the continuity of $\frac{\partial u(X, \theta)}{\partial X_{1}}$ and $\frac{\partial u(X, \theta)}{\partial X_{2}}$ in $X$ guarantees the existence, for each player, of a lower bound on the number of players for which the results hold. The continuity of the above derivatives in $\theta$ assures that a bound can be found independently from the set of players.

${ }^{6}$ With assumption 2, this implies that $\forall X_{2}, \forall \theta_{i} \frac{\partial u\left(X, \theta_{i}\right)}{\partial X_{1}} \gtreqless 0$ if $X_{1} \lesseqgtr \theta_{i 1}$ as well as $\forall X_{1}, \forall \theta_{i} \frac{\partial u\left(X, \theta_{i}\right)}{\partial X_{2}} \gtreqless 0$ if $X_{2} \lesseqgtr \theta_{i 2}$.

${ }^{7}$ Obviously, $\theta^{\Gamma}$ exists, by the Kakutani's fixed point theorem, and is unique, because $\forall j=1,2$

$$
\theta_{j}^{\prime}>\theta_{j} \Longrightarrow \max H_{j}\left(\theta^{\prime}\right) \leq \min H_{j}(\theta)
$$
}


Proposition 3 Under Assumption $4, \forall \varepsilon>0, \exists n_{0}$ such that $\forall n \geq n_{0}$ if $\sigma$ is a Nash equilibrium of a game $\Gamma$ with $n$ voters, then:

( $\alpha)$ if $\theta_{i} \leq X(\bar{\sigma})-\vec{\varepsilon}$ and $c \notin \underset{k \in M}{\operatorname{argmin}}\left[\alpha\left(\theta_{i}\right) \zeta_{k 1}+\zeta_{k 2}\right]$ then $\sigma_{i}^{c}=0$;

( $\beta)$ if $\theta_{i} \geq X(\bar{\sigma})+\vec{\varepsilon}$ and $c \notin \underset{k \in M}{\operatorname{argmax}}\left[\alpha\left(\theta_{i}\right) \zeta_{k 1}+\zeta_{k 2}\right]$ then $\sigma_{i}^{c}=0$;

$(\gamma)$ if $\theta_{i 1} \leq X_{1}(\bar{\sigma})-\varepsilon, \theta_{i 2} \geq X_{2}(\bar{\sigma})+\varepsilon$ and $c \notin \underset{k \in M}{\operatorname{argmin}}\left[\alpha\left(\theta_{i}\right) \zeta_{k 1}-\zeta_{k 2}\right]$ then $\sigma_{i}^{c}=0 ;$

( $\delta)$ if $\theta_{i 1} \geq X_{1}(\bar{\sigma})+\varepsilon, \theta_{i 2} \leq X_{2}(\bar{\sigma})-\varepsilon$ and $c \notin \underset{k \in M}{\operatorname{argmax}}\left[\alpha\left(\theta_{i}\right) \zeta_{k 1}-\zeta_{k 2}\right]$ then $\sigma_{i}^{c}=0$.

The above Proposition shows that, for large electorate, parties that are not located on the boundary of the convex hull of parties' positions will not take a relevant amount of votes.

\section{Conclusion}

In this short paper we have studied strategic voting under proportional representation, when the policy space is multidimensional. We prove that, when four parties located at the corners of the policy space exist, if voters' preferences are single-peaked in each dimension with the peak independent from the other dimension, and if the utility function is continuously differentiable with respect to the policy, there exist a "cutpoint" policy such that any equilibrium conforms to such a cutpoint, that is to say almost everybody located on the left-bottom of the cutpoint votes for the left-bottom corner and so on. Because the assumption that there are four parties located at the corners is quite strong, we drop it, at the cost to deal with a specific utility function. If the preferences are represented by a loss function that is a weighted sum of each issue's distance from the player's bliss policy, in any equilibrium, only parties located on the boundary of the convex hull of parties' positions take a relevant amount of votes.

\section{References}

[1] Austen-Smith D., and J. Banks (1988), "Elections, Coalitions, and Legislative Outcomes", The American Political Science Review, 82: 405-422.

[2] Baron D. P. and D. Diermeier (2001), "Elections, Governments, and Parliaments in Proportional Representation Systems", Quarterly Journal of Economics, 116: 933-967.

[3] De Sinopoli F., and G. Iannantuoni (2003), A Spatial Voting Model where Proportional Representation Leads to Two-Party Equilibria, CEIS Tor Vergata RPS No.3.

[4] Schofied N., and I. Sened (2002), Local Nash Equilibrium in Multiparty Politics, Annals of Operational Research, 109: 193-210. 


\section{Appendix: Proofs of Proposition 2 and 3}

Given a mixed strategy $\sigma_{j}$, the player $j$ 's vote is a random vector ${ }^{8} \tilde{s}_{j}$ with $\operatorname{Pr}\left(\tilde{s}_{j}=k\right)=\sigma_{j}^{k}$. Given $\sigma_{-i}=\left(\sigma_{1}, \ldots \sigma_{i-1}, \sigma_{i+1}, \ldots \sigma_{n}\right)$, let $\tilde{s}^{-i}=\frac{1}{n-1} \sum_{j \in N / i} \tilde{s}_{j}$ and $\bar{\sigma}^{-i}=\frac{1}{n-1} \sum_{j \in N / i} \sigma_{j}$. The following Lemma will be useful to prove both Propositions:

Lemma $4 \forall \phi>0$ and $\forall \delta>0$, if $n>\frac{m}{4 \phi^{2} \delta}+1$, then $\forall \sigma, \forall i$

$$
\operatorname{Pr}\left(\left|\tilde{s}^{-i}-\bar{\sigma}^{-i}\right| \leq \vec{\phi}\right)>1-\delta .
$$

Proof. To prove the lemma we can use Chebyshev's inequality component by component. Given $\sigma_{-i}$, it is easy to verify that $E\left(\tilde{s}_{j k}\right)=\sigma_{j}^{k}$ and $\operatorname{Var}\left(\tilde{s}_{j k}\right)=\sigma_{j}^{k}\left(1-\sigma_{j}^{k}\right) \leq \frac{1}{4}$, hence $E\left(\tilde{s}_{k}^{-i}\right)=\bar{\sigma}_{k}^{-i}$ and $\operatorname{Var}\left(\tilde{s}_{k}^{-i}\right) \leq \frac{1}{4(n-1)}$. By Chebyshev's inequality we know that $\forall k, \forall \phi$ :

$$
\operatorname{Pr}\left(\left|\tilde{s}_{k}^{-i}-\bar{\sigma}_{k}^{-i}\right|>\phi\right) \leq \frac{1}{4(n-1) \phi^{2}} .
$$

Hence

$$
\operatorname{Pr}\left(\left|\tilde{s}^{-i}-\bar{\sigma}^{-i}\right| \leq \vec{\phi}\right) \geq 1-\sum_{k} \operatorname{Pr}\left(\left|\tilde{s}_{k}^{-i}-\bar{\sigma}_{k}^{-i}\right|>\phi\right) \geq 1-\frac{m}{4(n-1) \phi^{2}},
$$

which is strictly greater than $1-\delta$ for $n>\frac{m}{4 \phi^{2} \delta}+1$.

Proof of Proposition 2.

The first step of the proof consists in showing:

Lemma 5 Under Assumptions 1-3, $\forall \varepsilon>0, \exists n_{0}^{L B}$ such that $\forall n \geq n_{0}^{L B}$, if the game has $n$ voters and if $\theta_{i} \leq X(\bar{\sigma})-\vec{\varepsilon}$, then $L B$ is the only best reply for player $i$ to $\sigma_{-i}$.

Proof. Fix $\varepsilon>0$. Define, $\forall \theta \in\left[0,1-\frac{\varepsilon}{2}\right]^{2}$,

$$
M_{\varepsilon 1}(\theta)=\max _{\left(X_{1}, X_{2}\right) \in\left[\theta+\frac{\varepsilon}{2}, 1\right]^{2}} \frac{\partial u(X, \theta)}{\partial X_{1}} .
$$

By Assumption 2 we know that $M_{\varepsilon 1}(\theta)<0$. Moreover, given the continuity of $\frac{\partial u(X, \theta)}{\partial X_{1}}$, we can apply the theorem of the maximum to deduce that the function $M_{\varepsilon 1}(\theta)$ is continuous, hence it has a maximum on $\left[0,1-\frac{\varepsilon}{2}\right]^{2}$, which is strictly negative. Let

$$
M_{\varepsilon 1}^{*}=\max _{\theta \in\left[0,1-\frac{\varepsilon}{2}\right]^{2}} M_{\varepsilon 1}(\theta) .
$$

Define analogously $M_{\varepsilon 2}^{*}$. Let $M_{\varepsilon}^{*}=\max \left\{M_{\varepsilon 1}^{*}, M_{\varepsilon 2}^{*}\right\}$, and $\underline{\mathrm{c}}=\min _{k \in M /\{L B\}}\left\{\zeta_{k 1}+\zeta_{k 2}\right\}$. Let $\bar{M}_{1}$ and $\bar{M}_{2}$ denote, respectively, the upper bound ${ }^{9}$ of $\left|\frac{\partial u(X, \theta)}{\partial X_{1}}\right|$ and of $\left|\frac{\partial u(X, \theta)}{\partial X_{2}}\right|$ on $\mathbb{X} \times \not \subset, \bar{M}=\max \left\{\bar{M}_{1}, \bar{M}_{2}\right\}$ and let $\delta_{\varepsilon}^{*}=\frac{-M_{\varepsilon}^{*} \mathrm{C}}{2 \bar{M}-M_{\varepsilon}^{*} \underline{\mathrm{C}}}$ and $\phi^{*}=\frac{(-2+\sqrt{6}) \varepsilon}{m}$. We prove that if $n>\frac{m}{4 \phi^{* 2} \delta_{\varepsilon}^{*}}+1$, then $L B$ is the only best reply for player $i$, which, setting $n_{0}^{L B}$ equal to the smallest integer strictly greater than $\frac{m}{4 \phi^{* 2} \delta_{\varepsilon}^{*}}+1$, directly implies the claim.

Take a party $c \neq L B$. We will show that $n>\frac{m}{4 \phi^{* 2} \delta_{\varepsilon}^{*}}+1$ implies

$$
U_{i}\left(\sigma_{-i}, c\right)-U_{i}\left(\sigma_{-i}, L B\right)<0
$$

and, hence, $c \neq L B$ is not a best reply for player $i$.

$$
U_{i}\left(\sigma_{-i}, c\right)-U_{i}\left(\sigma_{-i}, L B\right)=\sum_{s_{-i} \in S_{-i}} \sigma\left(s_{-i}\right)\left[u\left(X\left(s_{-i}, c\right)-\frac{1}{n}\left(\zeta_{c}-\zeta_{L B}\right), \theta_{i}\right)-u\left(X\left(s_{-i}, c\right), \theta_{i}\right)\right] .
$$

\footnotetext{
${ }^{8}$ We remind readers that a vote is a vector with $m$ components.

${ }^{9}$ The continuity of $\frac{\partial u(X, \theta)}{\partial X_{1}}$ and $\frac{\partial u(X, \theta)}{\partial X_{2}}$ assures that these bounds exist.
} 
Because the outcome function $X(s)$ depends only upon $v(s)$, denoting with $V^{-i}$ the set of all vectors representing the share of votes obtained by each party with $(n-1)$ voters, the right-hand side of the above expression can be written as:

$$
\sum_{v^{-i} \in V^{-i}} \operatorname{Pr}\left(\tilde{s}^{-i}=v^{-i}\right)\left[u\left(X\left(v^{-i}, c\right)-\frac{1}{n}\left(\zeta_{c}-\zeta_{L B}\right), \theta_{i}\right)-u\left(X\left(v^{-i}, c\right), \theta_{i}\right)\right]
$$

where, with abuse of notation, $X\left(v^{-i}, c\right)=\frac{\zeta_{c}}{n}+\frac{n-1}{n} \sum_{k=1}^{m} \zeta_{k} v_{k}^{-i}$.

By the mean value theorem we know that $\forall v^{-i}$,

$\exists X^{*}$ belonging to the line joining $X\left(v^{-i}, c\right)-\frac{1}{n} \zeta_{c}$ and $X\left(v^{-i}, c\right)$ such that

$$
\frac{\left[u\left(X\left(v^{-i}, c\right), \theta_{i}\right)-u\left(X\left(v^{-i}, c\right)-\frac{1}{n}\left(\zeta_{c}-\zeta_{L B}\right), \theta_{i}\right)\right]}{\frac{1}{n}}=\frac{\partial u\left(X^{*}, \theta_{i}\right)}{\partial X_{1}} \zeta_{c 1}+\frac{\partial u\left(X^{*}, \theta_{i}\right)}{\partial X_{2}} \zeta_{c 2} .
$$

Hence we have:

$$
\begin{gathered}
U_{i}\left(\sigma_{-i}, c\right)-U_{i}\left(\sigma_{-i}, L B\right)=\frac{1}{n} \sum_{v^{-i} \in V^{-i}} \operatorname{Pr}\left(\tilde{s}^{-i}=v^{-i}\right) \frac{\left[u\left(X\left(v^{-i}, c\right), \theta_{i}\right)-u\left(X\left(v^{-i}, c\right)-\frac{1}{n}\left(\zeta_{c}-\zeta_{L B}\right), \theta_{i}\right)\right]}{\frac{1}{n}} \\
\leq \frac{1}{n} \operatorname{Pr}\left(\left|\tilde{s}^{-i}-\bar{\sigma}^{-i}\right| \leq \vec{\phi}^{*}\right)\left[M_{n 1}^{*}\left(\phi^{*}, \theta_{i 1}\right) \zeta_{c 1}+M_{n 2}^{*}\left(\phi^{*}, \theta_{i 2}\right) \zeta_{c 2}\right]+\frac{2}{n}\left(1-\operatorname{Pr}\left(\left|\tilde{s}_{k}^{-i}-\bar{\sigma}^{-i}\right| \leq \vec{\phi}^{*}\right)\right) \bar{M}
\end{gathered}
$$

where

$$
\begin{aligned}
M_{n 1}^{*}\left(\phi^{*}, \theta_{i 1}\right) & =\max _{\left(X_{1}, X_{2}\right) \in\left[X\left(\bar{\sigma}^{-i}-\vec{\phi}^{*}, c\right)-\frac{1}{n} \zeta_{c}, 1\right]^{2}} \frac{\partial u\left(X, \theta_{i}\right)}{\partial X_{1}} \\
M_{n 2}^{*}\left(\phi^{*}, \theta_{i 2}\right) & =\max _{\left(X_{1}, X_{2}\right) \in\left[X\left(\bar{\sigma}^{-i}-\vec{\phi}^{*}, c\right)-\frac{1}{n} \zeta_{c}, 1\right]^{2}} \frac{\partial u\left(X, \theta_{i}\right)}{\partial X_{2}} .
\end{aligned}
$$

Now we prove that, for $n>\frac{m}{4 \phi^{*} \delta_{\varepsilon}^{*}}+1, M_{n 1}^{*}\left(\phi^{*}, \theta_{i 1}\right) \leq M_{\varepsilon}^{*}$ as well as $M_{n 2}^{*}\left(\phi^{*}, \theta_{i 2}\right) \leq M_{\varepsilon}^{*}$.

We show that $M_{n 1}^{*}\left(\phi^{*}, \theta_{i 1}\right) \leq M_{\varepsilon}^{*}$, the other one being completely analogous. From the definition of $M_{\varepsilon}^{*}$, it suffices to prove that $M_{n 1}^{*}\left(\phi^{*}, \theta_{i 1}\right) \leq M_{\varepsilon 1}$, which is true if $X_{1}\left(\bar{\sigma}^{-i}-\vec{\phi}^{*}, c\right)-\frac{1}{n} \zeta_{c 1}$ is greater than $\theta_{i 1}+\frac{\varepsilon}{2}$, and $X_{2}\left(\bar{\sigma}^{-i}-\vec{\phi}^{*}, c\right)-\frac{1}{n} \zeta_{c 2}$ is greater than $\theta_{i 2}+\frac{\varepsilon}{2}$. We only prove the inequality for the first coordinate, the proof for the second coordinate being similar.

$$
\begin{gathered}
X_{1}\left(\bar{\sigma}^{-i}-\vec{\phi}^{*}, c\right)-\frac{1}{n} \zeta_{c 1}=\frac{n-1}{n} \sum_{k} \bar{\sigma}_{k}^{-i} \zeta_{k 1}-\frac{n-1}{n} \sum_{k} \phi^{*} \zeta_{k 1}= \\
X_{1}(\bar{\sigma})-\frac{1}{n} \sum_{k} \sigma_{i}^{k} \zeta_{k 1}-\frac{n-1}{n} \sum_{k} \phi^{*} \zeta_{k 1}>X_{1}(\bar{\sigma})-\frac{1}{n}-m \phi^{*} \geq \theta_{i 1}+\varepsilon-\frac{1}{n}-m \phi^{*} .
\end{gathered}
$$

Hence, this step of the proof is concluded by noticing that $\delta_{\varepsilon}^{*}$ is by definition less than $\frac{1}{2}$, hence ${ }^{10}$

$$
\begin{gathered}
\theta_{i 1}+\varepsilon-\frac{1}{n}-m \phi^{*}>\theta_{i 1}+\varepsilon-m \phi^{*}-\frac{2 \phi^{* 2}}{m}=\theta_{i 1}+\varepsilon-\frac{(20-8 \sqrt{6}) \varepsilon^{2}}{m^{3}}-\varepsilon(-2+\sqrt{6}) \geq \\
\theta_{i 1}+\varepsilon\left(1-\frac{(20-8 \sqrt{6})}{8}+2-\sqrt{6}\right)=\theta_{i 1}+\frac{1}{2} \varepsilon .
\end{gathered}
$$

By Lemma 4, we know that, for $n>\frac{m}{4 \phi^{* 2} \delta_{\varepsilon}^{*}}+1$,

$$
\operatorname{Pr}\left(\left|\tilde{s}^{-i}-\bar{\sigma}^{-i}\right| \leq \vec{\phi}^{*}\right)\left[M_{n 1}^{*}\left(\phi^{*}, \theta_{i 1}\right) \zeta_{c 1}+M_{n 2}^{*}\left(\phi^{*}, \theta_{i 2}\right) \zeta_{c 2}\right]+2\left(1-\operatorname{Pr}\left(\left|\tilde{s}^{-i}-\bar{\sigma}^{-i}\right| \leq \vec{\phi}^{*}\right)\right) \bar{M}<
$$

\footnotetext{
${ }^{10}$ In the following we assume that $\varepsilon \leq 1$, since otherwise the proposition is trivially true.
} 


$$
\left(1-\delta_{\varepsilon}^{*}\right) M_{\varepsilon}^{*} \underline{\mathrm{c}}+2 \delta_{\varepsilon}^{*} M=\left(1-\frac{-M_{\varepsilon}^{*} \underline{\mathrm{c}}}{2 M-M_{\varepsilon}^{*} \underline{\mathrm{c}}}\right) M_{\varepsilon}^{*} \underline{\mathrm{c}}+2 \frac{-M_{\varepsilon}^{*} \underline{\mathrm{c}}}{2 M-M_{\varepsilon}^{*} \underline{\mathrm{c}}} M=0
$$

which concludes the proof.

A symmetric argument proves:

Lemma 6 Under Assumptions 1-3, $\forall \varepsilon>0, \exists n_{0}^{R T}, n_{0}^{L T}, n_{0}^{R B}$ such that:

( $\alpha) \forall n \geq n_{0}^{R T}$, if the game has $n$ voters and if $\theta_{i} \geq X(\bar{\sigma})+\vec{\varepsilon}$, then $R T$ is the only best reply for player $i$ to $\sigma_{-i}$.

( $\beta) \forall n \geq n_{0}^{L T}$, if the game has $n$ voters and if $\theta_{i 1} \leq X_{1}(\bar{\sigma})-\varepsilon$, and $\theta_{i 2} \geq X_{2}(\bar{\sigma})+\varepsilon$, then LT is the only best reply for player $i$ to $\sigma_{-i}$.

$(\gamma) \forall n \geq n_{0}^{R B}$, if the game has $n$ voters and if $\theta_{i 1} \geq X_{1}(\bar{\sigma})+\varepsilon$, and $\theta_{i 2} \leq X_{2}(\bar{\sigma})-\varepsilon$, then $R B$ is the only best reply for player $i$ to $\sigma_{-i}$.

Combining the result of the following Lemma, we obtain Proposition 2

Lemma 7 Under Assumptions 1-3, $\forall \eta>0, \exists n_{0}$ such that $\forall n \geq n_{0}$ if $\sigma$ is a Nash equilibrium of a game $\Gamma$ with $n$ voters, then:

( $\alpha$ ) if $\theta_{i 1} \leq \theta_{1}^{\Gamma}-\eta$ then $\sigma_{i} \in\{L B, L T\}$

(B) if $\theta_{i 1} \geq \theta_{1}^{\Gamma}+\eta$ then $\sigma_{i} \in\{R B, R T\}$

$(\gamma)$ if $\theta_{i 2} \leq \theta_{2}^{\Gamma}-\eta$ then $\sigma_{i} \in\{L B, R B\}$

(ס) if $\theta_{i 2} \geq \theta_{2}^{\Gamma}+\eta$ then $\sigma_{i} \in\{L T, R T\}$.

Proof. We only prove $(\alpha)$ and $(\beta)$ because the proof of $(\gamma)$ and $(\delta)$ is completely similar. Fix $\eta$ and, in Lemmas 5 and 6 , take $\varepsilon=\frac{\eta}{2}$ and let $n_{0}$ be the greater number among the corresponding $n_{0}^{L B}, n_{0}^{R T}, n_{0}^{L T}$, and $n_{0}^{R B}$. It is easy to see that if $n \geq n_{0}$ and $\sigma$ is a Nash equilibrium of $\Gamma, \theta_{1}^{\Gamma}-\frac{\eta}{2} \leq X_{1}(\bar{\sigma}) \leq \theta_{1}^{\Gamma}+\frac{\eta}{2}$. Suppose by contradiction that $\theta_{1}^{\Gamma}-\frac{\eta}{2}>X_{1}(\bar{\sigma})$, then Lemma 6 implies that all voters $i$ with $\theta_{i 1} \geq \theta_{1}^{\Gamma}$ vote either for $R B$ or for $R T$ and hence $\theta_{1}^{\Gamma} \leq X_{1}(\bar{\sigma})$, contradicting $\theta_{1}^{\Gamma}-\frac{\eta}{2}>X_{1}(\bar{\sigma})$. Analogously for the second inequality. Hence $\theta_{1}^{\Gamma}-\eta \leq X_{1}(\bar{\sigma})-\frac{\eta}{2}$ and $\theta_{1}^{\Gamma}-\eta \geq X_{1}(\bar{\sigma})+\frac{\eta}{2}$, which with Lemmas 5 and 6 complete the proof.

Proof of Proposition 3: We first prove the following Lemma:

Lemma 8 Under Assumption $4, \forall \varepsilon>0, \exists n_{0}^{L B}$ such that $\forall n \geq n_{0}^{L B}$, if the game has $n$ voters, $\sigma$ is a Nash equilibrium, $\theta_{i} \leq X(\bar{\sigma})-\vec{\varepsilon}$, and $c \notin \underset{k \in M}{\operatorname{argmin}}\left[\alpha\left(\theta_{i}\right) \zeta_{k 1}+\zeta_{k 2}\right]$ then $\sigma_{i}^{c}=0$.

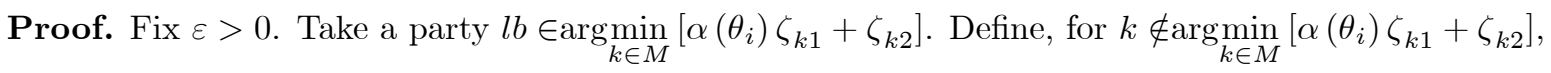

$$
D^{k}\left(\theta_{i}\right)=\left[\alpha\left(\theta_{i}\right) \zeta_{l b 1}+\zeta_{l b 2}-\alpha\left(\theta_{i}\right) \zeta_{k 1}-\zeta_{k 2}\right]
$$

Define also $D\left(\theta_{i}\right)=\max _{k \notin \arg \min _{k \in M}\left[\alpha\left(\theta_{i}\right) \zeta_{k 1}+\zeta_{k 2}\right]} D^{k}\left(\theta_{i}\right)$, as well as $D=\max _{\theta_{i}} D\left(\theta_{i}\right)$. Clearly $D$ is strictly negative.

Let $\alpha^{*}=\max _{\theta} \alpha(\theta), \delta^{*}=\frac{-D}{1+\alpha^{*}-D}$, and $\phi^{*}=\frac{(-2+2 \sqrt{2}) \varepsilon}{m}$. Clearly $\delta^{*}$ and $\phi^{*}$ are strictly positive.

Now we prove that if $n>\frac{m}{4 \phi^{* 2} \delta^{*}}+1$, then $\forall c \notin \underset{k \in M}{\arg \min }\left[\alpha\left(\theta_{i}\right) \zeta_{k 1}+\zeta_{k 2}\right]$ :

$$
U_{i}\left(\sigma_{-i}, c\right)-U_{i}\left(\sigma_{-i}, l b\right)<0
$$

which, setting $n_{0}^{L B}$ as the smallest integer greater or equal to $\frac{m}{4 \phi^{* 2} \delta^{*}}+1$, implies the claim.

As in the proof of Lemma 5 ,

$$
U_{i}\left(\sigma_{-i}, c\right)-U_{i}\left(\sigma_{-i}, l b\right)=\sum_{v^{-i} \in V^{-i}} \operatorname{Pr}\left(\tilde{s}^{-i}=v^{-i}\right)\left[u\left(X\left(v^{-i}, c\right), \theta_{i}\right)-u\left(X\left(v^{-i}, l b\right), \theta_{i}\right)\right] .
$$


Notice that, if

$$
\left\{\begin{array}{l}
\theta_{i 1} \leq \min \left\{X_{1}\left(s_{-i}, c\right), X_{1}\left(s_{-i}, l b\right)\right\} \\
\theta_{i 2} \leq \min \left\{X_{2}\left(s_{-i}, c\right), X_{2}\left(s_{-i}, l b\right)\right\}
\end{array}\right.
$$

then

$$
u\left(X\left(s_{-i}, c\right), \theta_{i}\right)-u\left(X\left(s_{-i}, l b\right), \theta_{i}\right)=\frac{1}{n}\left[\alpha\left(\theta_{i}\right) \zeta_{l b 1}+\zeta_{l b 2}-\alpha\left(\theta_{i}\right) \zeta_{c 1}-\zeta_{c 2}\right]=\frac{1}{n} D^{c}\left(\theta_{i}\right)
$$

Moreover,

$$
\max _{s_{-i}, c}\left|u\left(X\left(s_{-i}, c\right), \theta_{i}\right)-u\left(X\left(s_{-i}, l b\right), \theta_{i}\right)\right| \leq \frac{1}{n}\left[\alpha\left(\theta_{i}\right)+1\right] .
$$

Obviously, $X_{1}\left(s_{-i}, \overrightarrow{0}\right) \leq \min \left\{X_{1}\left(s_{-i}, c\right), X_{1}\left(s_{-i}, l b\right)\right\}$, as well as $X_{2}\left(s_{-i}, \overrightarrow{0}\right) \leq \min \left\{X_{2}\left(s_{-i}, c\right), X_{2}\left(s_{-i}, l b\right)\right\} .{ }^{11}$ From Lemma 4 we know, for $n>\frac{m}{4 \phi^{* 2} \delta^{*}}+1$, that $\operatorname{Pr}\left(\left|\tilde{s}^{-i}-\bar{\sigma}^{-i}\right| \leq \vec{\phi}^{*}\right)>1-\delta^{*}$. Because, for $n>\frac{m}{4 \phi^{* 2} \delta^{*}}+1$, $X\left(\bar{\sigma}^{-i}-\phi^{*}, \overrightarrow{0}\right)>X(\bar{\sigma})-\vec{\varepsilon} \geq \theta_{i}{ }^{12}$, we can deduce:

$$
\begin{aligned}
u\left(X\left(\sigma_{-i}, c\right), \theta_{i}\right)-u\left(X\left(\sigma_{-i}, l b\right), \theta_{i}\right) & =\sum_{v^{-i} \in V^{-i}} \operatorname{Pr}\left(\overline{\tilde{s}}^{-i}=v^{-i}\right)\left[u\left(X\left(v^{-i}, c\right), \theta_{i}\right)-u\left(X\left(v^{-i}, l b\right), \theta_{i}\right)\right]< \\
\frac{1}{n}\left[\left(1-\delta^{*}\right) D+\delta^{*}\left(1+\alpha^{*}\right)\right] & =\frac{1}{n}\left[\left(1-\frac{-D}{1+\alpha^{*}-D}\right) D+\frac{-D}{1+\alpha^{*}-D}\left(1+\alpha^{*}\right)\right]=0
\end{aligned}
$$

and, hence, $c \notin \underset{k \in M}{\arg \min }\left[\alpha\left(\theta_{i}\right) \zeta_{k 1}+\zeta_{k 2}\right]$ cannot be a best reply for player $i$.

Analogously, the following Lemma can be proved:

Lemma 9 Under Assumption 4, $\forall \varepsilon>0, \exists n_{0}^{R T}, n_{0}^{L T}, n_{0}^{R B}$ such that $(\alpha) \forall n \geq n_{0}^{R T}$, if the game has $n$ voters, $\sigma$ is a Nash equilibrium, $\theta_{i} \geq X(\bar{\sigma})+\vec{\varepsilon}$, and $c \notin \underset{k \in M}{\operatorname{argmax}}\left[\alpha\left(\theta_{i}\right) \zeta_{k 1}+\zeta_{k 2}\right]$ then $\sigma_{i}^{c}=0$.

( $\beta) \forall n \geq n_{0}^{L T}$, if the game has $n$ voters, $\sigma$ is a Nash equilibrium, $\theta_{i 1} \leq X_{1}(\bar{\sigma})-\varepsilon, \theta_{i 2} \geq X_{2}(\bar{\sigma})+\varepsilon$, and $c \notin \underset{k \in M}{\operatorname{argmin}}\left[\alpha\left(\theta_{i}\right) \zeta_{k 1}-\zeta_{k 2}\right]$ then $\sigma_{i}^{c}=0$.

$(\gamma) \forall n \geq n_{0}^{R B}$, if the game has $n$ voters, $\sigma$ is a Nash equilibrium, $\theta_{i 1} \geq X_{1}(\bar{\sigma})+\varepsilon, \theta_{i 2} \leq X_{2}(\bar{\sigma})-\varepsilon$, and $c \notin \underset{k \in M}{\operatorname{argmax}}\left[\alpha\left(\theta_{i}\right) \zeta_{k 1}-\zeta_{k 2}\right]$ then $\sigma_{i}^{c}=0$.

Setting $n_{0}=\max \left\{n_{0}^{L B}, n_{0}^{R T}, n_{0}^{L T}, n_{0}^{R B}\right\}$ completes the proof.

\footnotetext{
${ }^{11}$ With abuse of notation $X\left(s_{-i}, \overrightarrow{0}\right)$ denotes the outcome that would have been resulted if a party in $(0,0)$ existed and player $i$ voted for it.

${ }^{12} \mathrm{We}$ only prove the inequality for the first coordinate because the proof for the second coordinate is completely analogous.

$$
X_{1}\left(\bar{\sigma}^{-i}-\vec{\phi}^{*}, \overrightarrow{0}\right)=X_{1}(\bar{\sigma})-\frac{1}{n} \sum_{k} \sigma_{i}^{k} \zeta_{k 1}-\frac{n-1}{n} \sum_{k} \phi^{*} \zeta_{k 1}>X_{1}(\bar{\sigma})-\frac{1}{n}-m \phi^{*}
$$
}

This step of the proof is concluded by noticing that $\delta_{\varepsilon}^{*}$ is by definition less than $\frac{1}{2}$, hence

$$
X_{1}(\bar{\sigma})-\frac{1}{n}-m \phi^{*}>X_{1}(\bar{\sigma})-m \phi^{*}-\frac{2 \phi^{* 2}}{m}=X_{1}(\bar{\sigma})-(-2+2 \sqrt{2}) \varepsilon-\frac{24-16 \sqrt{2}}{m^{3}} \varepsilon^{2}>X_{1}(\bar{\sigma})-\varepsilon
$$




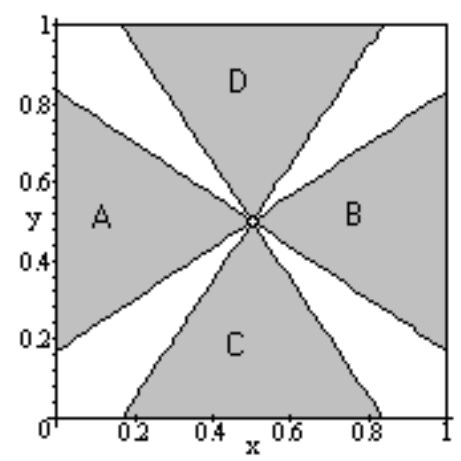

Figure 1: $x=\theta_{1}, y=\theta_{2}$.

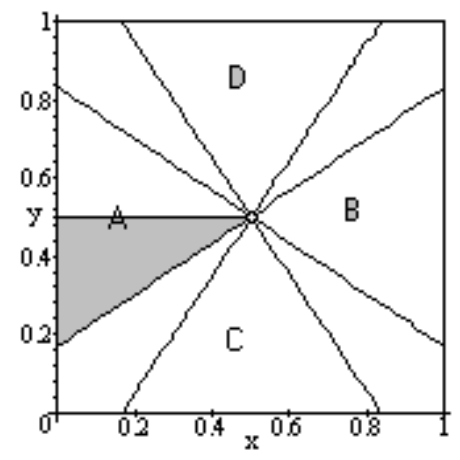

Figure 2: $x=\theta_{1}, y=\theta_{2}$. 